\title{
Revisiting Batchelor's theory of two-dimensional turbulence
}

\author{
DAVID G. DRITSCHEL, CHUONG V. TRAN \\ AND RICHARD K. SCOTT \\ School of Mathematics and Statistics, University of St Andrews, St Andrews KY16 9SS, UK
}

(Received 26 July 2006 and in revised form 13 July 2007)

Recent mathematical results have shown that a central assumption in the theory of two-dimensional turbulence proposed by Batchelor (Phys. Fluids, vol. 12, 1969, p. 233) is false. That theory, which predicts a $\chi^{2 / 3} k^{-1}$ enstrophy spectrum in the inertial range of freely-decaying turbulence, and which has evidently been successful in describing certain aspects of numerical simulations at high Reynolds numbers $R e$, assumes that there is a finite, non-zero enstrophy dissipation $\chi$ in the limit of infinite $R e$. This, however, is not true for flows having finite vorticity. The enstrophy dissipation in fact vanishes.

We revisit Batchelor's theory and propose a simple modification of it to ensure vanishing $\chi$ in the limit $R e \rightarrow \infty$. Our proposal is supported by high Reynolds number simulations which confirm that $\chi$ decays like $1 / \ln R e$, and which, following the time of peak enstrophy dissipation, exhibit enstrophy spectra containing an increasing proportion of the total enstrophy $\left\langle\omega^{2}\right\rangle / 2$ in the inertial range as $R e$ increases. Together with the mathematical analysis of vanishing $\chi$, these observations motivate a straightforward and, indeed, alarmingly simple modification of Batchelor's theory: just replace Batchelor's enstrophy spectrum $\chi^{2 / 3} k^{-1}$ with $\left\langle\omega^{2}\right\rangle k^{-1}(\ln R e)^{-1}$.

\section{Introduction}

Recently, two distinct approaches to the problem of enstrophy dissipation in two-dimensional turbulence have reached the conclusion that, for finite vorticity, the enstrophy dissipation vanishes in the inviscid limit, $v \rightarrow 0$. The approach of Lopes Filho, Mazzucato \& Nussenzveig Lopes (2006), applicable to finite times only, has its roots in the mathematical analyses of DiPerna \& Majda (1987), DiPerna \& Lions (1989) and Eyink (2001), who exploited the convergence of Navier-Stokes solutions to their Euler counterparts under certain conditions on the vorticity field, in particular the condition of bounded enstrophy (mean-square vorticity). The approach of Tran \& Dritschel (2006), applied basic inequalities to the evolution of palinstrophy, or the mean-square vorticity gradient, and assumed power-law spectra for the enstrophy inertial range. This approach provides the upper bound on the enstrophy dissipation

$$
v\left\langle|\nabla \omega|^{2}\right\rangle \leqslant \frac{\|\omega\|_{\infty}\left\langle\omega^{2}\right\rangle}{(\ln R e)^{1 / 2}},
$$

which is valid uniformly in time. Here $\langle\cdot\rangle$ denotes the domain average, $\omega$ is the vorticity, $\|\omega\|_{\infty}$ is its supremum, $\left\langle\omega^{2}\right\rangle$ is (twice) the enstrophy and $R e$ is an appropriate Reynolds number. 
This result is in apparent contradiction to the phenomenological scaling theory of two-dimensional turbulence proposed by Batchelor (1969), as that theory uses the anticipated finite, non-zero value of the enstrophy dissipation $\chi(t)$ in the limit $R e \rightarrow \infty$ (or $v \rightarrow 0$ ) as a key parameter. By dimensional analysis, Batchelor concluded that the enstrophy spectrum in fully developed turbulence will acquire the self-similar form $\Omega(k)=\alpha \chi^{2 / 3} k^{-1}$ (where $\alpha$ is a dimensionless constant) over a range of wavenumbers $k$ extending from the 'energy-containing' scales, say around wavenumber $k_{0}$, to the viscous scales, say around $k_{v} \sim v^{-1 / 2}-$ more precisely over the range $k_{0} \ll k \ll k_{v}$, the so-called 'inertial range'.

Batchelor's theory has been extensively examined (see Kida, Yamada \& Ohkitani 1988, Bartello \& Warn 1996, Chasnov 1997, Davidson 2004 and many others) and recent high-resolution numerical results strongly support the $k^{-1}$ form of the inertialrange enstrophy spectrum (see Dmitruk \& Montgomery 2005 and references therein). Although there is still disagreement between theory and numerical simulations with regard to the temporal scaling of the enstrophy decay at late times, predicted as $t^{-2}$ but observed numerically as approximately $t^{-1}$ (e.g. Chasnov 1997), there seems little doubt that certain aspects of Batchelor's theory are correct, particularly during early stages when turbulent enstrophy dissipation is largest. Yet, what is the implication of the false assumption of finite, non-zero enstrophy dissipation in the limit $R e \rightarrow \infty$ ? The purpose of this paper is to show that this assumption is, in fact, not crucial. There appears to be a simple remedy.

The plan of the paper is as follows. In the next section we briefly recall the results of Tran \& Dritschel (2006), principally to clarify the role of integral quantities in the production and dissipation of enstrophy $\frac{1}{2}\left\langle\omega^{2}\right\rangle$ and palinstrophy $\frac{1}{2}\left\langle|\nabla \omega|^{2}\right\rangle$. In $\S 3$, these and other quantities are monitored in a series of high Reynolds number numerical simulations of unforced turbulence, starting from smooth initial conditions. Our results are consistent with our analytically-derived upper bound (1.1) as well as with previous numerical results of Dmitruk \& Montgomery (2005), who first pointed out the tendency for the enstrophy dissipation $v\left\langle|\nabla \omega|^{2}\right\rangle$ to decay with $R e$ - apparently as $(\ln R e)^{-1}$. A careful analysis of other integral quantities leads us to the conclusion that there is, in fact, no $R e$-independent measure of dissipation in two-dimensional turbulence. On the other hand - and this is the important point - such a measure does not appear to be required in Batchelor's scaling theory. Instead, in $\S 4$ we demonstrate that Batchelor's scaling arguments go through virtually unchanged if we use the enstrophy itself in place of the enstrophy dissipation. Indeed, this modification is already evident in the mathematical analysis presented in Tran \& Dritschel (2006). Furthermore, we propose that dissipation in two-dimensional turbulence and related quasigeostrophic geophysical turbulence is benign - a coarse-grained sampling of a perfectly inviscid turbulent flow would result in an apparent decay of enstrophy similar to that observed in the Navier-Stokes equations.

\section{Production and dissipation in turbulence}

We begin with the vorticity-streamfunction form of the two-dimensional NavierStokes equations,

$$
\omega_{t}+J(\psi, \omega)=v \Delta \omega, \quad \Delta \psi=\omega,
$$

where $J(\psi, \omega)=\psi_{x} \omega_{y}-\psi_{y} \omega_{x}$ and subscripts denote differentiation. We consider a doubly-periodic domain, and importantly restrict our attention to flows having bounded vorticity - these arguably being physically realistic. In other words, $\|\omega\|_{\infty}$, the 
supremum of vorticity, is taken to be finite and independent of $v$ as $v \rightarrow 0$. This is not, we emphasize, a significant restriction, as there is a wide class of non-trivial Euler flows (with $v=0$ ) having bounded vorticity - for instance flows having piecewiseuniform vorticity (described by 'contour dynamics', see Zabusky, Hughes \& Roberts 1979; Dritschel 1989 and references therein). Such Euler flows conserve $\omega$ pointwise (following fluid 'particles'), implying $\|\omega\|_{\infty}$ is invariant as well as the conservation of a generally infinite number of 'Casimirs' $\langle F(\omega)\rangle$, where $F$ is any functional of $\omega$ (e.g. $F=\frac{1}{2} \omega^{2}$ for enstrophy). Euler flows also conserve energy $\frac{1}{2}\left\langle|\nabla \psi|^{2}\right\rangle$.

Under the action of viscosity, $\|\omega\|_{\infty}$, the energy and all convex Casimirs (satisfying $\left.F^{\prime \prime}>0\right)$ strictly decay in time. In particular, the enstrophy satisifies the equation

$$
\frac{1}{2} \frac{\mathrm{d}}{\mathrm{d} t}\left\langle\omega^{2}\right\rangle=-v\left\langle|\nabla \omega|^{2}\right\rangle
$$

The behaviour of the enstrophy dissipation therefore depends on the palinstrophy evolution,

$$
\frac{1}{2} \frac{\mathrm{d}}{\mathrm{d} t}\left\langle|\nabla \omega|^{2}\right\rangle=\langle\Delta \omega J(\psi, \omega)\rangle-v\left\langle|\Delta \omega|^{2}\right\rangle .
$$

Using basic inequalities, Tran \& Dritschel (2006) showed that the growth of palinstrophy is bounded by

$$
\frac{1}{2} \frac{\mathrm{d}}{\mathrm{d} t}\left\langle|\nabla \omega|^{2}\right\rangle \leqslant \frac{\left\langle|\Delta \omega|^{2}\right\rangle}{\left\langle|\nabla \omega|^{2}\right\rangle}\left(\|\omega\|_{\infty}\left\langle\omega^{2}\right\rangle^{1 / 2} \frac{\left\langle|\nabla \omega|^{2}\right\rangle}{\left\langle|\Delta \omega|^{2}\right\rangle^{1 / 2}}-v\left\langle|\nabla \omega|^{2}\right\rangle\right) .
$$

Note the enstrophy dissipation (see (2.2)) appears explicitly within the brackets. Since the maximum enstrophy dissipation occurs at the end of a period of palinstrophy growth, it follows that the enstrophy dissipation is bounded from above by the first term in the brackets, i.e.

$$
v\left\langle|\nabla \omega|^{2}\right\rangle \leqslant\|\omega\|_{\infty}\left\langle\omega^{2}\right\rangle^{1 / 2} \frac{\left\langle|\nabla \omega|^{2}\right\rangle}{\left\langle|\Delta \omega|^{2}\right\rangle^{1 / 2}} .
$$

For flows possessing finite energy and enstrophy, Tran \& Shepherd (2002) have proved that the centroid wavenumber $s \equiv\left\langle\omega^{2}\right\rangle^{1 / 2} /\left\langle|\nabla \psi|^{2}\right\rangle^{1 / 2}$ decreases in time, implying that the enstrophy spectrum cannot become shallower than $C k^{-1}$ - Batchelor's spectrum in the limit $R e \rightarrow \infty$. But for this spectrum, as well as any steeper spectrum, it is simple to show that the right-hand side of (2.5) vanishes in this limit.

\section{Numerical simulations}

The results of five primary simulations of (2.1) are examined next. The numerical method is a conventional pseudospectral one with the time step chosen for numerical stability and the viscosity chosen to ensure that quantities in the palinstrophy evolution equation (2.3), in particular $\Delta \omega$, are accurately resolved.

The time integration is performed in spectral space, using an integrating factor to incorporate the viscous term exactly, and a fourth-order Runge-Kutta scheme. The time step is chosen to be $0.0025(512 / n)$, where $n$ is the spatial resolution (number of grid points in $x$ and in $y$ ). This time step is less than half the CFL time step required for stability at the initial time (and never rises above $75 \%$ of the CFL time step during the flow evolution). In tests using resolutions up to $n=2048$, halving the time step had no measurable impact on solution accuracy.

As in Dmitruk \& Montgomery (2005), we begin with a flow having unit r.m.s. velocity, or energy $\frac{1}{2}\left\langle|\nabla \psi|^{2}\right\rangle=\frac{1}{2}$ (the maximum velocity is about 2.3). Its initial energy 
spectrum takes the form $E(k)=a k^{8} \exp \left[-8 \pi(k / 8)^{2}\right]$, which peaks at $k=\sqrt{32 / \pi} \approx 3.2$ and is more than $10^{36}$ times smaller by $k=32$. While such a spectrum is already peaked at low wavenumbers, no significant inverse energy cascade takes place over the short duration of our simulations. Similarly broad initial conditions were used previously by Kida et al. (1988) and Dmitruk \& Montgomery (2005) for the purpose of quantifying enstrophy dissipation.

The viscosity is chosen so that the approximate viscous wavenumber occurs at $3 / 4$ of the maximum wavenumber $n / 2$. Specifically, we take $\nu=4 \pi /(3 n / 8)^{2}$, where $4 \pi$ is approximately equal to the initial peak vorticity, $\|\omega\|_{\infty}(0)=11.941946$. By trial and error, this choice was found to ensure adequate dissipation at high wavenumbers to resolve the statistics of $\left\langle|\Delta \omega|^{2}\right\rangle$ and other fine-scale quantities.

We define the initial Reynolds number in terms of the initial energy-enstrophy centroid wavenumber $k_{0}=\left\langle\omega^{2}\right\rangle^{1 / 2} /\left\langle|\nabla \psi|^{2}\right\rangle^{1 / 2}=3.541$, the root-mean-square vorticity $\left\langle\omega^{2}\right\rangle^{1 / 2}=3.541$ and $v$ by the relation $\operatorname{Re} \equiv\left\langle\omega^{2}\right\rangle^{1 / 2} / \nu k_{0}^{2}=\left\langle|\nabla \psi|^{2}\right\rangle / \nu\left\langle\omega^{2}\right\rangle^{1 / 2}$. Then, for the five simulations, we have $R e=8.28 \times 10^{2}, 3.31 \times 10^{3}, 1.33 \times 10^{4}, 5.30 \times 10^{4}$, and $2.12 \times 10^{5}$.

To minimize aliasing errors, we followed the approach of Hou \& Li (2006), who have demonstrated the advantages of spectral filtering over traditional de-aliasing. When $x$ or $y$ derivatives are taken in spectral space, they are multiplied by the spectral filter $\rho(2 k / n) \equiv \exp \left(-\alpha(2 k / n)^{m}\right)$ with $\alpha=m=36$, where $k=k_{x}$ or $k_{y}$. This allows one to retain about $12-15 \%$ more modes than the standard $2 / 3$ dealiasing rule. The only observable impact of this filter is on numerical stability: without it, erroneous growth occurs at the highest wavenumbers due to the improper account of nonlinearity there.

We examine five spatial resolutions, $n=512,1024,2048,4096$ and 8192. These appear to be high enough to examine the nature of dissipation in two-dimensional turbulence, as the results below indicate. The five cases were integrated past the peak in palinstrophy (at which time spectra indicate that the turbulence is fully developed, see below) and until the palinstrophy falls to half of its peak value.

Images of the vorticity field for three of the resolutions are shown in figure 1. The times shown correspond to when the palinstrophy is growing and is roughly half its peak value (left), when it is at its peak value (middle), and when it is decaying and is again half its peak value (right). As expected, with decreasing viscosity there is substantially more fine structure, especially at and beyond the palinstrophy peak. Note that the peak palinstrophy time increases with Reynolds number, approximately as $\ln R e$ (see below), consistent with classical views of the enstrophy cascade (see Rhines 1975; Kida et al. 1988; Davidson 2004; Dmitruk \& Montgomery 2005 and others). This is shown more explicitly below.

We next turn to quantitative analyses. The palinstrophy spectra, $k^{4} E(k, t)=$ $k^{2} \Omega(k, t)$, for $n=1024,2048,4096,8192$, and at various times are shown in figure 2. Increasingly, a range close to the classical $k^{1}$ spectrum develops, but clearly a wide range of scales is required to capture it. The spectrum has filled out completely by the palinstrophy peak, and afterward the spectrum at moderate to high wavenumbers decays while preserving its basic shape. In fact, at these times, the high- $k$ end of the spectrum is well fitted by an exponential decay of the form $k^{2} \Omega(k, t) \propto k \exp \left(-b k / k_{v}\right)$, with $b \approx 5.4$ and $k_{v} \equiv k_{0} \operatorname{Re}^{1 / 2}\left(=\left\langle\omega^{2}\right\rangle^{1 / 4} \nu^{-1 / 2}\right)$, where $k_{0}$ is the instantaneous energyenstrophy centroid wavenumber - see figure 3 . The same decay was found by Kida et al. (1988) for strikingly different initial conditions, and moreover the value of $b$ is numerically comparable. It is meaningful, therefore, to say that the turbulence is 'fully developed' by the time the palinstrophy reaches its maximum. 

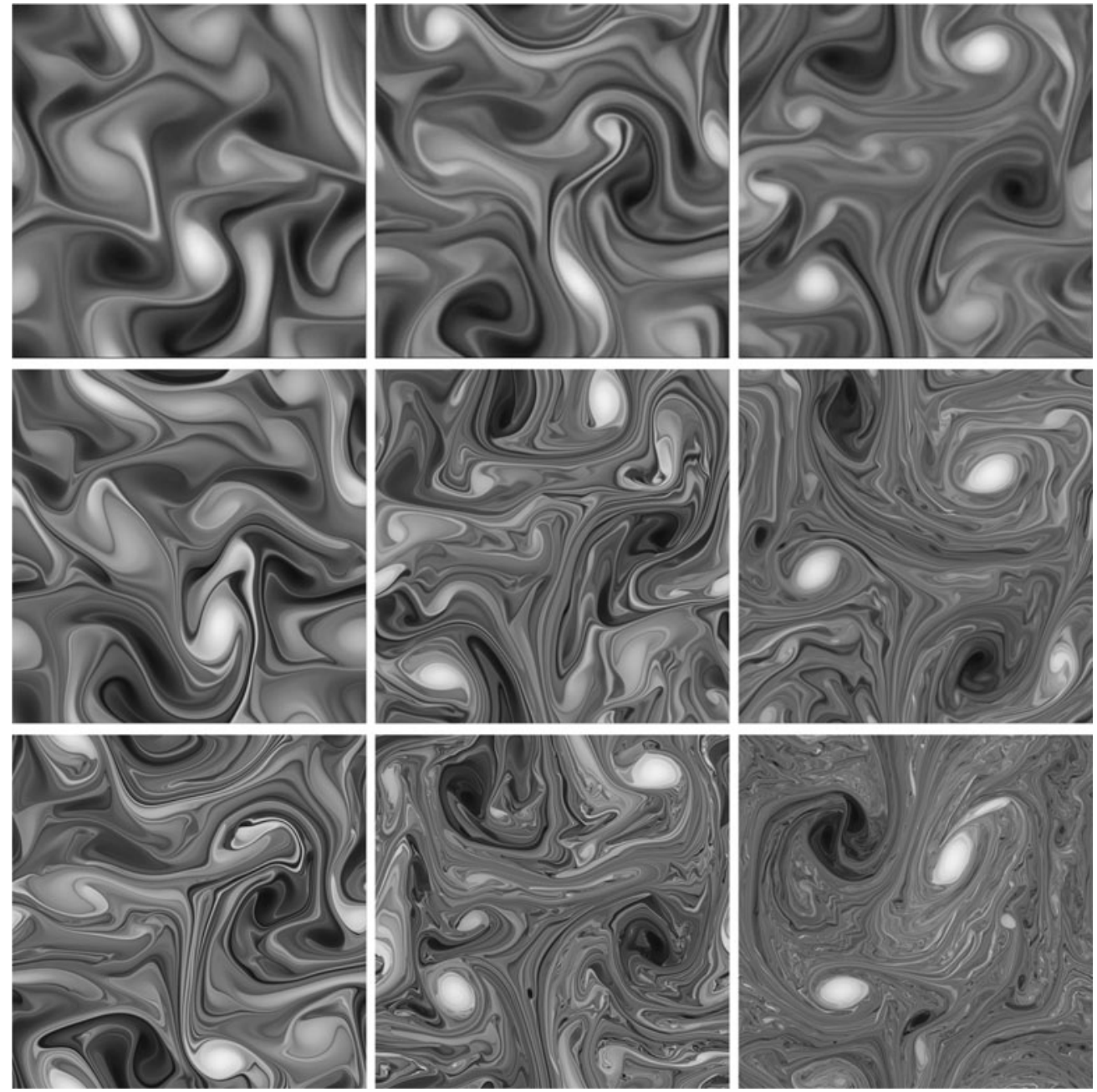

Figure 1. Evolution of the vorticity field $\omega$ in the simulations with $n=512$ (first row), 2048 (second row) and 8192 (third row). The times shown are $t=2.2,4.2$ and 7.6 (first row); $t=3.2$, 6.4 and 12.8 (second row); and $t=5.0,9.0$ and 19.0 (third row).

We now examine the evolution of various integral quantities. Figure 4 shows the energy $\frac{1}{2}\left\langle|\nabla \psi|^{2}\right\rangle$, enstrophy $\frac{1}{2}\left\langle\omega^{2}\right\rangle$, enstrophy dissipation $v\left\langle|\nabla \omega|^{2}\right\rangle$, vorticity supremum $\|\omega\|_{\infty}$ and $v\left\langle|\Delta \omega|^{2}\right\rangle^{1 / 2}$ for the four highest resolutions. The Euler invariants are progressively better conserved with increasing $R e$, as expected. At $n=8192$, the energy decreases by $0.050 \%$ by the end of the simulation, and $\|\omega\|_{\infty}$ decreases by only $0.037 \%$. On the other hand, $\frac{1}{2}\left\langle\omega^{2}\right\rangle$ decreases by $54 \%$. The enstrophy dissipation $v\left\langle|\nabla \omega|^{2}\right\rangle$ together with $v\left\langle|\Delta \omega|^{2}\right\rangle^{1 / 2}$ grows rapidly initially through nonlinear interactions, reaches a peak or a double peak, then finally decays.

If we compare the different Reynolds numbers, we see that the enstrophy dissipation does indeed diminish with $R e$. Figure 5(a) shows $v\left\langle|\nabla \omega|^{2}\right\rangle$ for the five cases versus time, and figure $5(b)$ shows enstrophy dissipation sculed by a linear polynomial in $\ln R e$, specifically $a_{0}+a_{1} \ln R e$, versus scaled time $t /\left(a_{0}+a_{1} \ln R e\right)$. The coefficients $a_{0}=-0.440$ and $a_{1}=0.300$ were obtained by a least-square fit of the peak enstrophy dissipation in our five simulations, and in four additional ones with half the viscosity at resolutions 

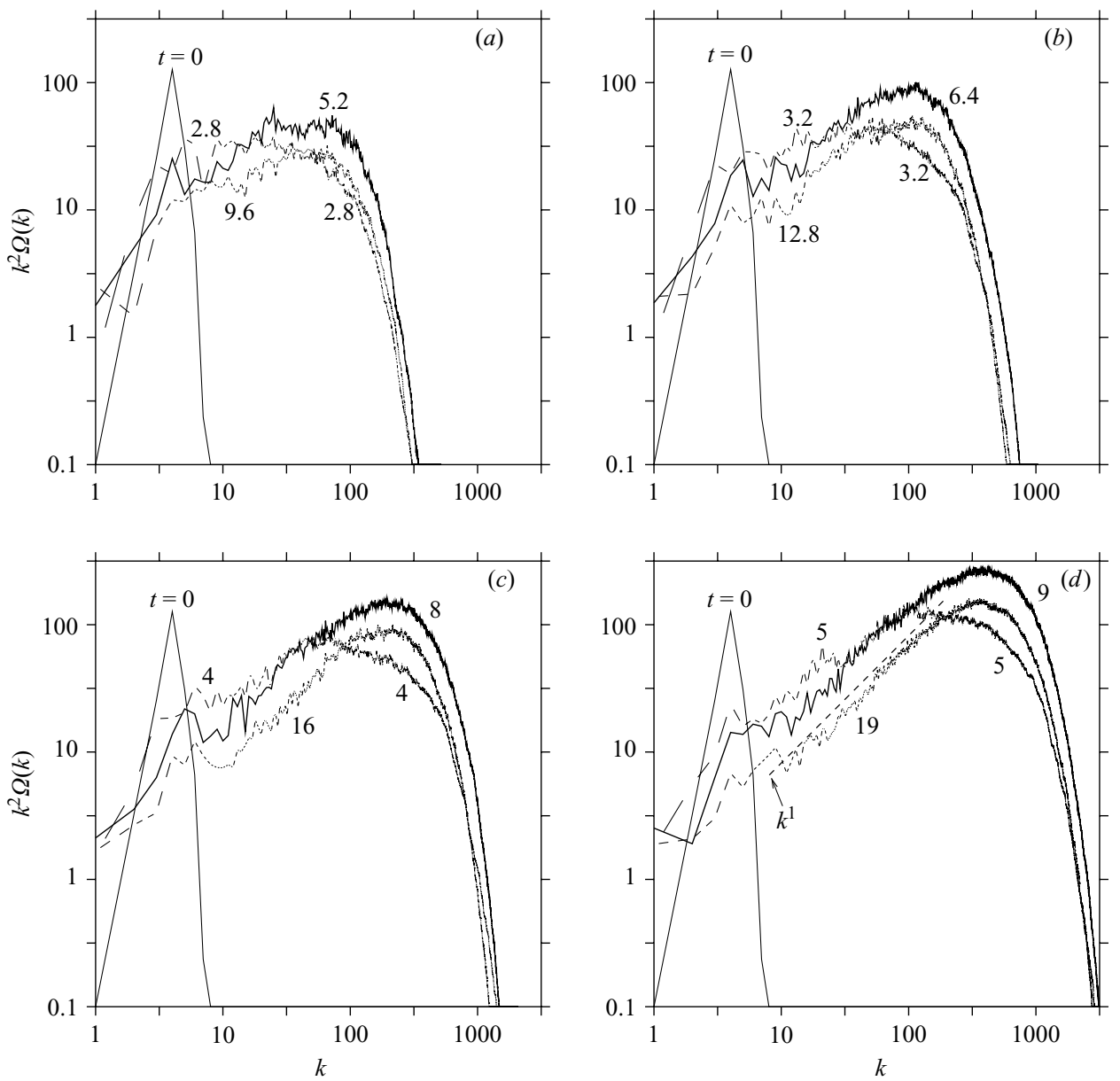

FIGURE 2. Palinstrophy spectra $k^{2} \Omega(k, t)$ at $t=0$ and at times when the palinstrophy is half its peak value (and growing), at its peak, and half its peak (and decaying). The panels show $n=1024(a), 2048(b), 4096(c), 8192(d)$. A reference slope of $k^{1}$ is shown as a thick dashed line $(d)$.

512 through 4096. Note that the scaling approximately aligns the peaks in enstrophy dissipation. These results are consistent with those of Dmitruk \& Montgomery (2005), who also found that the peak enstrophy dissipation scales as $(\ln R e)^{-1}$.

This decay with $R e$ is significantly stronger than that suggested by the analytical upper bound (2.5), which, for the classical Batchelor spectrum reduces to

$$
v\left\langle|\nabla \omega|^{2}\right\rangle \leqslant \frac{\|\omega\|_{\infty}\left\langle\omega^{2}\right\rangle}{(\ln R e)^{1 / 2}}
$$

(see Tran \& Dritschel 2006). Of course (3.1) is an upper bound only, and the inequalities used in its derivation could be generous. A steeper dependence on $R e$ is not ruled out. In fact, if we use an alternative estimate of the palinstrophy production term in (2.3), namely

$$
\begin{aligned}
\langle\Delta \omega J(\psi, \omega)\rangle & =-\left\langle\omega_{x} J\left(\psi_{x}, \omega\right)\right\rangle-\left\langle\omega_{y} J\left(\psi_{y}, \omega\right)\right\rangle \\
& =\left\langle\left(\omega_{x}^{2}-\omega_{y}^{2}\right) \psi_{x y}\right\rangle+\left\langle\omega_{x} \omega_{y}\left(\psi_{y y}-\psi_{x x}\right)\right\rangle \\
& \leqslant 2\|S\|_{\infty}\left\langle|\nabla \omega|^{2}\right\rangle
\end{aligned}
$$



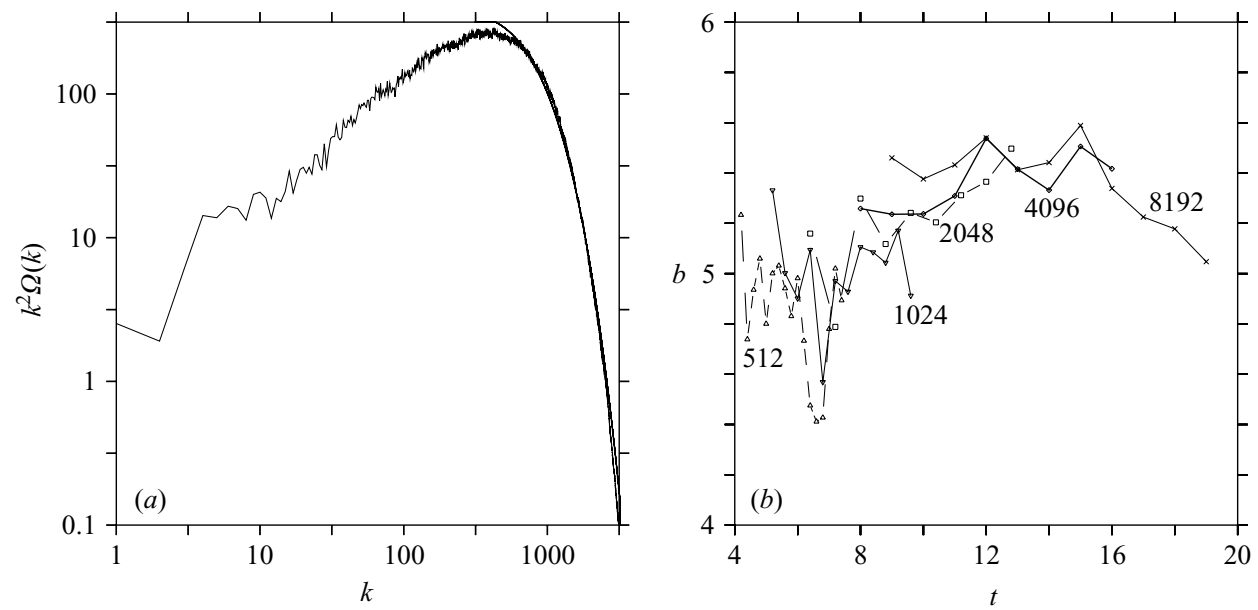

FIGURE 3. Palinstrophy spectra $k^{2} \Omega(k, t)$ for the 8192 simulation at $t=9$ (thin curve) and its least-squares fit to $C k \exp \left(-b k / k_{v}\right)$ over $0.2 k_{v} \leqslant k \leqslant 1.6 k_{v}$ (bold curve). (b) The coefficient $b$ as a function of time for $n=512$ (dashed line plus triangles), $n=1024$ (thin line plus inverted triangles), $n=2048$ (dashed line plus squares), $n=4096$ (bold line plus diamonds), and $n=8192$ (thin line plus crosses). Only times at and beyond the peak palinstrophy time are examined.

where $S=\left|\psi_{x x}-\psi_{y y}\right| / 2+\left|\psi_{x y}\right|$ is the irrotational strain, then we have

$$
\frac{1}{2} \frac{\mathrm{d}}{\mathrm{d} t}\left\langle|\nabla \omega|^{2}\right\rangle \leqslant \frac{\left\langle|\Delta \omega|^{2}\right\rangle}{\left\langle|\nabla \omega|^{2}\right\rangle}\left(2\|S\|_{\infty} \frac{\left\langle|\nabla \omega|^{2}\right\rangle^{2}}{\left\langle|\Delta \omega|^{2}\right\rangle}-v\left\langle|\nabla \omega|^{2}\right\rangle\right)
$$

For Batchelor's spectrum, this implies

$$
v\left\langle|\nabla \omega|^{2}\right\rangle \leqslant \frac{2\|S\|_{\infty}\left\langle\omega^{2}\right\rangle}{\ln R e} .
$$

This appears promising, but unfortunately we cannot bound the strain norm $\|S\|_{\infty}$ in terms of the Euler invariants, and furthermore our results (see figure 6) suggest that $\|S\|_{\infty}$ increases with $R e$, possibly as $\ln R e$.

Note, however, that the increasing time taken to reach the peak enstrophy dissipation with increasing $R e$ appears to compensate for the lower peak dissipation, giving a finite, almost Reynolds-number-independent net dissipation by this time see figure 7. In figure 7, the symbols on the enstrophy curves mark the time of peak enstrophy dissipation. If we plot the enstrophy curves versus $t^{*} \equiv t+\ln (v)$, they cross at nearly the same point (not shown), but with a tendency for slightly less net dissipation with increasing $R e$. Whether or not this dissipation ultimately vanishes as $R e \rightarrow \infty$ cannot yet be decided. Similar behaviour was found by Dmitruk $\&$ Montogomery (2005) for independent initial conditions.

Figure 8 shows $v\left\langle|\Delta \omega|^{2}\right\rangle^{1 / 2}$ and the scaled quantity $v\left(\left\langle|\Delta \omega|^{2}\right\rangle\left(a_{0}+a_{1} \ln R e\right)\right)^{1 / 2}$ in the same format as figure 5 , and using the same values of the coefficients $a_{0}$ and $a_{1}$. Again, in scaled time, the curves tend to align, but notice that their peak values (on the left) diminish significantly less rapidly with increasing $R e$ compared to $v\left\langle|\nabla \omega|^{2}\right\rangle$ in figure 5 . This is consistent with the analytical estimate (2.5) for Batchelor's spectrum (3.1), for which the ratio $\left\langle|\Delta \omega|^{2}\right\rangle^{1 / 2} /\left\langle|\nabla \omega|^{2}\right\rangle$ diverges as $R e \rightarrow \infty$.

On the other hand, the ratio $\left\langle|\Delta \omega|^{2}\right\rangle /\left\langle|\nabla \omega|^{2}\right\rangle$ should be proportional to $k_{v}^{2} \propto v^{-1}$, for enstrophy spectra shallower than $k^{-3}$ (see Tran \& Dritschel 2006). That is, this ratio defines the upper limit of the 'inertial range', and it is expected to increase with 

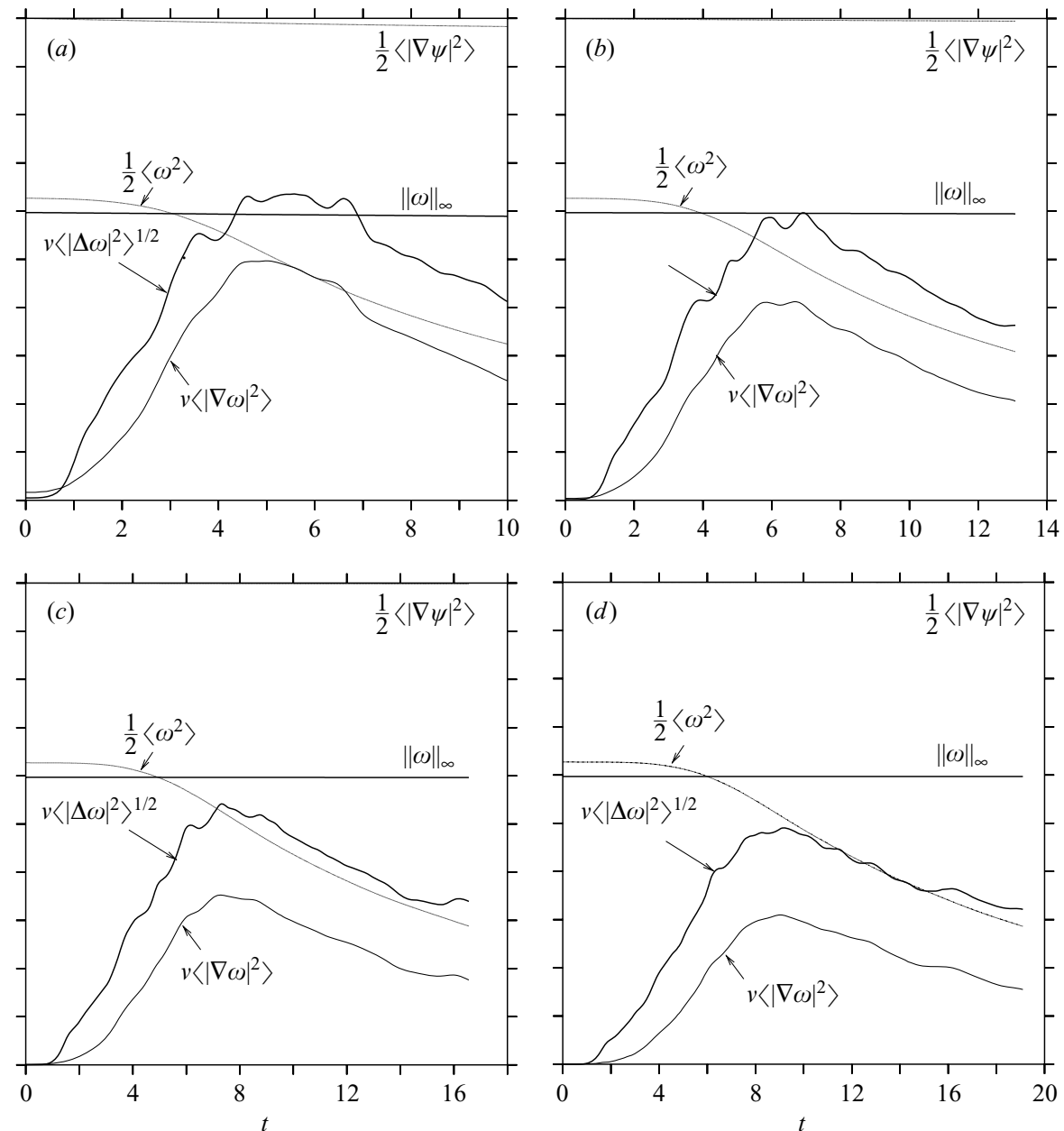

FIGURE 4. Composite evolution of various norms for the simulations with $n=1024(a), 2048$ (b), $4096(c)$, and $8192(d)$. The maximum plot scales are 0.5 for $\frac{1}{2}\left\langle|\nabla \psi|^{2}\right\rangle, 10$ for $\frac{1}{2}\left\langle\omega^{2}\right\rangle, 20$ for $\|\omega\|_{\infty}$, and 1 for $v\left\langle|\nabla \omega|^{2}\right\rangle$ and $v\left\langle|\Delta \omega|^{2}\right\rangle^{1 / 2}$. The $y$ axis is linear for all quantities.

$R e$. For Batchelor's spectrum, including an exponential decay for large $k$ (see figure 3 and Kida et al. 1988), namely $\Omega(k) \sim C k^{-1} \exp \left(-b k / k_{v}\right)$, elementary calculations show that $\left\langle|\Delta \omega|^{2}\right\rangle /\left\langle|\nabla \omega|^{2}\right\rangle \approx 6\left(k_{\nu} / b\right)^{2}$. If we use this expression to define $b$, then

$$
b^{2}=\frac{6\left\langle\omega^{2}\right\rangle^{1 / 2}\left\langle|\nabla \omega|^{2}\right\rangle}{v\left\langle|\Delta \omega|^{2}\right\rangle},
$$

where we have substituted $k_{v}=\left\langle\omega^{2}\right\rangle^{1 / 4} v^{-1 / 2}$. The time-averaged values of $b$ for the five simulations are listed in table 1 , for times at and beyond the palinstrophy peak. These values compare well with those found by fitting the palinstrophy spectra in figure $3(b)$, independently verifying the (expected) growth of the inertial range with $R e$. The implication is that the effective dissipation wavenumber is $k_{v} / b$, i.e. considerably less than the order-of-magnitude estimate $k_{v}=\left\langle\omega^{2}\right\rangle^{1 / 4} v^{-1 / 2}$, yet still strongly divergent in the limit $v \rightarrow 0$. For the highest resolution simulation with $n=8192, k_{v} / b$ ranges from 254 to 285 , less than a tenth of the maximum wavenumber (4096) available to 

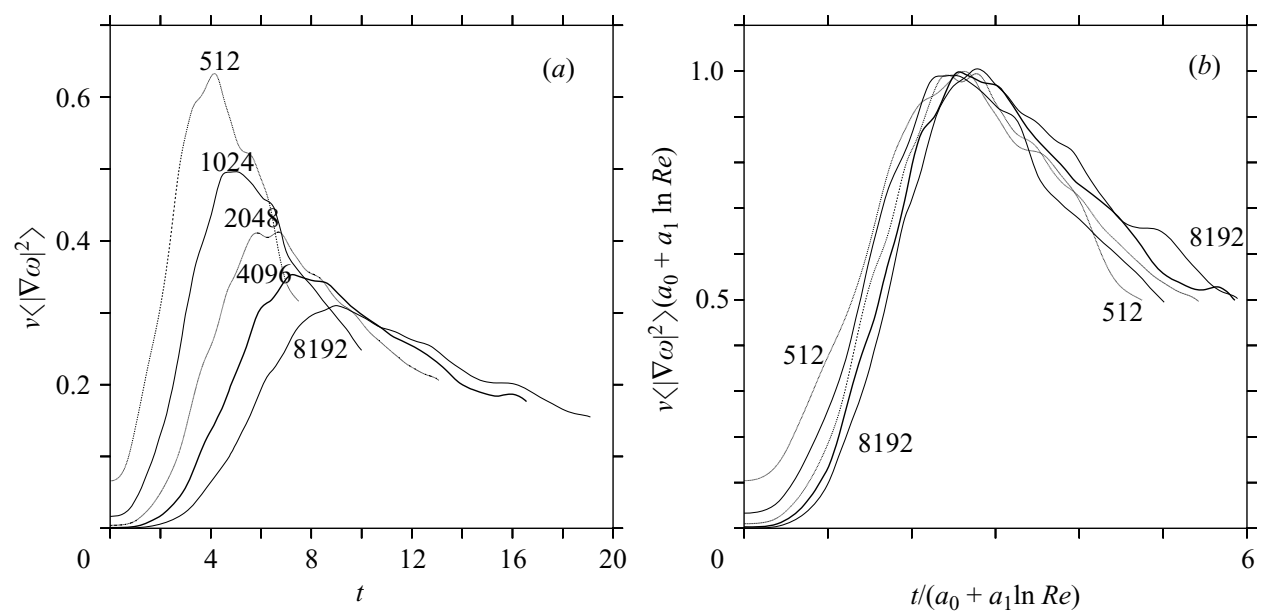

FIGURE 5. Enstrophy dissipation $v\left\langle|\nabla \omega|^{2}\right\rangle$ versus time $t(a)$ and scaled enstrophy dissipation $v\left\langle|\nabla \omega|^{2}\right\rangle\left(a_{0}+a_{1} \ln R e\right)$ versus scaled time $t /\left(a_{0}+a_{1} \ln R e\right)(b)$, with $n=512$ (short dashed), $n=1024$ (thin), $n=2048$ (long dashed), $n=4096$ (bold), and $n=8192$ (thin). Note, $a_{0}=-0.440$ and $a_{1}=0.300$.

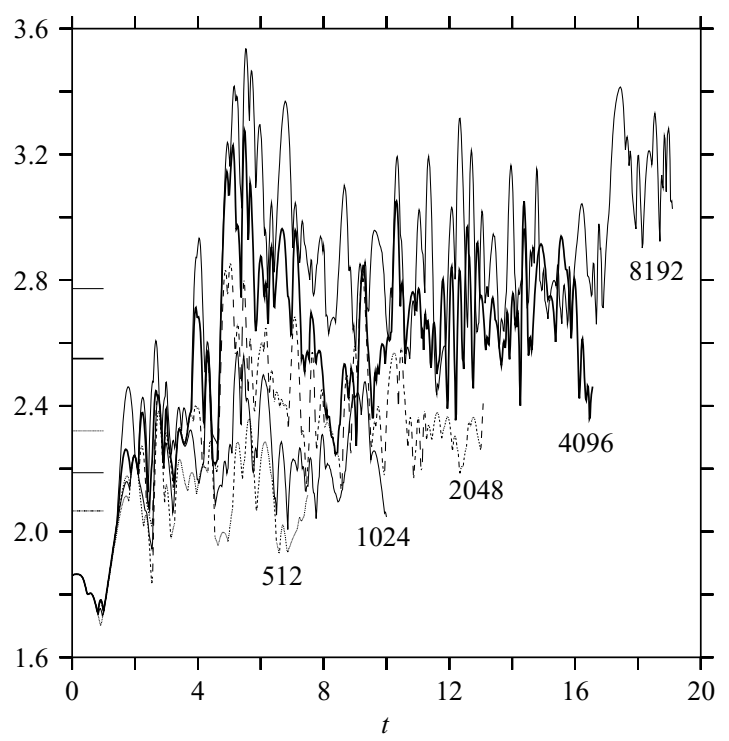

FIGURE 6. Ratio of strain supremum to root-mean-square vorticity, compared across resolution or $R e: n=512$ (short dashed), $n=1024$ (thin), $n=2048$ (long dashed), $n=4096$ (bold), and $n=8192$ (thin). The short lines on the left indicate the time averages.

the numerical simulation. While it is possible to reduce $v$ further while maintaining numerical stability, accuracy is seriously compromised for values of $v$ more than a factor of 2 smaller than those we have chosen.

\section{Concluding remarks}

In summary, we have examined a variety of quantities which might characterize dissipation in freely-decaying two-dimensional turbulence, combinations of energy 


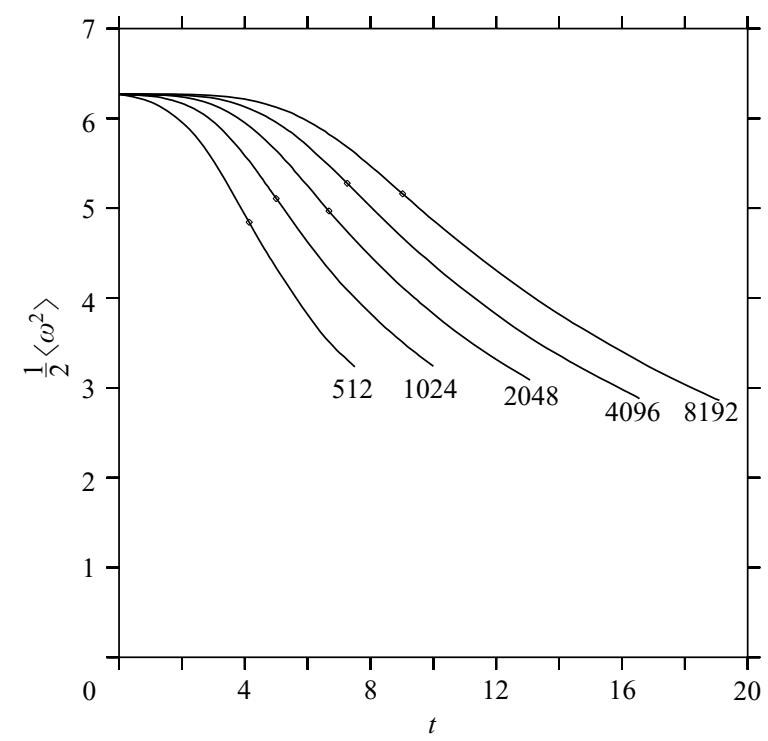

FIGURE 7. Enstrophy $\frac{1}{2}\left\langle\omega^{2}\right\rangle$, compared across resolution or Re, with the peak enstrophy dissipation times indicated.
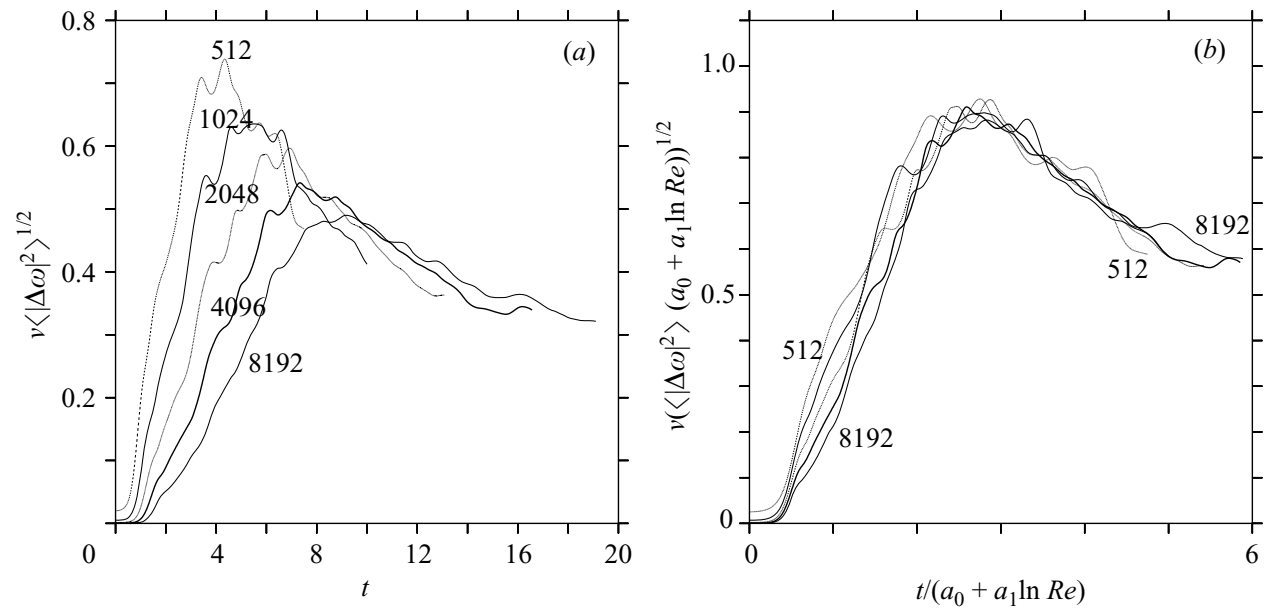

FIGURE 8. $v\left\langle|\Delta \omega|^{2}\right\rangle^{1 / 2}$ versus time $t(a)$ and $v\left(\left\langle|\Delta \omega|^{2}\right\rangle\left(a_{0}+a_{1} \ln R e\right)\right)^{1 / 2}$ versus scaled time $t /\left(a_{0}+a_{1} \ln R e\right)(b)$, with $n=512$ (short dashed), $n=1024$ (thin), $n=2048$ (long dashed), $n=4096$ (bold), and $n=8192$ (thin). Note, $a_{0}=-0.440$ and $a_{1}=0.300$.

Resolution, $n \quad$ Time average, $b \quad$ St. dev.

$\begin{array}{rll}512 & 4.6001 & 0.1456 \\ 1024 & 4.6723 & 0.0963 \\ 2048 & 4.7910 & 0.0866 \\ 4096 & 4.9048 & 0.0711 \\ 8192 & 4.9287 & 0.1114\end{array}$

TABLE 1. Average and standard deviation of the decay exponent $b$ calculated from $b=\sqrt{6} k_{v}\left[\left\langle|\nabla \omega|^{2}\right\rangle /\left\langle|\Delta \omega|^{2}\right\rangle\right]^{1 / 2}$ for the five resolutions. The statistics are computed for times at and beyond the time of peak palinstrophy. 


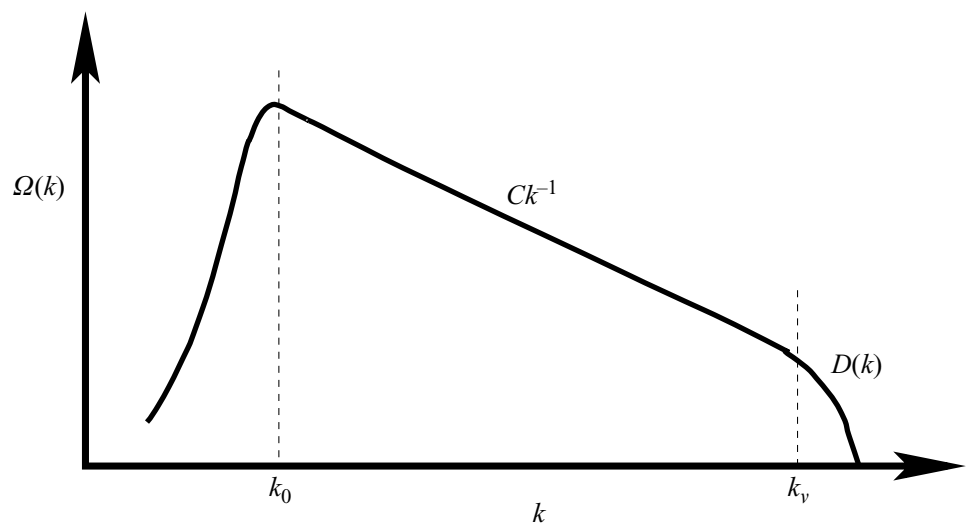

FIGURE 9. Hypothetical enstrophy spectrum $\Omega(k)$ exhibiting Batchelor's $k^{-1}$ inertial range between the energy-enstrophy wavenumber $k_{0}$ and the dissipation wavenumber $k_{v}$. The spectrum $D(k)$ for $k>k_{\nu}$ is here only required to be steeper than $k^{-1}$, but numerical simulations indicate it has an exponential form - see figure 3(a) and Kida et al. (1988). As Re increases, the inertial range $k_{0}<k<k_{v}$ contains an ever-increasing proportion of the total enstrophy, while the low-wavenumber range $k<k_{0}$ contains an increasing proportion of the total energy.

$\frac{1}{2}\left\langle|\nabla \psi|^{2}\right\rangle$, enstrophy $\frac{1}{2}\left\langle\omega^{2}\right\rangle$, enstrophy dissipation $v\left\langle|\nabla \omega|^{2}\right\rangle$, vorticity supremum $\|\omega\|_{\infty}$, strain supremum $\left\|\left|\psi_{x x}-\psi_{y y}\right| / 2+\left|\psi_{x y}\right|\right\|_{\infty}$, and $v\left\langle|\Delta \omega|^{2}\right\rangle^{1 / 2}$, and none appears to be Reynolds number independent. What our results and many others indicate is that an approximately self-similar inertial range with $\Omega(k) \approx C k^{-1}$ develops shortly after the time of peak enstrophy dissipation, as originally envisioned by Batchelor (1969). That much appears to be solid. Batchelor then used dimensional analysis to estimate $C$, and hypothesized that $C$, as well as the upper limit of the inertial range $k_{v}$, must be built from quantities characterizing the small-scale statistical structure of turbulence, just as in Kolmogorov's three-dimensional theory.

Batchelor used the viscosity $v\left[L^{2} T^{-1}\right]$ and the enstrophy dissipation $\chi\left[T^{-3}\right]$ (the parallel of energy dissipation in three-dimensional turbulence) to propose $C \propto \chi^{2 / 3}$ and $k_{v} \propto \chi^{1 / 3} v^{-1 / 2}$. However, the fact that $\chi$ vanishes in the inviscid limit $v \rightarrow 0$ (subject only to reasonable constraints of bounded vorticity and finite energy) means we need to look for a replacement for $\chi$. In fact, nothing in Batchelor's theory compels one to use $\chi$.

What we propose instead is alarmingly simple. It is based on the few solid pieces of evidence available, namely the continued extension of the inertial range $k_{0}<k<k_{v}$ with decreasing $v$ or increasing Re. Referring to figure 9, which illustrates a hypothetical enstrophy spectrum $\Omega(k)$, it is evident that the low- and highwavenumber tails of the spectrum, i.e. $k<k_{0}$ and $k>k_{\nu}$, contribute negligibly to the total enstrophy as $R e \rightarrow \infty$. This follows because $k_{0}$ does not grow with $R e$, and in the dissipation range $D(k)$ is steeper than $k^{-1}$. These are reasonable assumptions. In this case, as in Tran \& Dritschel (2006), we may assign essentially all of the enstrophy to the inertial range, leading to

$$
\frac{1}{2}\left\langle\omega^{2}\right\rangle \approx \int_{k_{0}}^{k_{v}} C k^{-1} \mathrm{~d} k=C \ln \left(k_{\nu} / k_{0}\right) .
$$

Hence, we are led to the simple result $C \approx\left\langle\omega^{2}\right\rangle / 2 \ln \left(k_{\nu} / k_{0}\right)=\left\langle\omega^{2}\right\rangle / \ln R e$ using our definition of $R e$. Batchelor was aware of this result (see the discussion following (9) in Batchelor 1969), but it did not persuade him to change his scaling theory. 

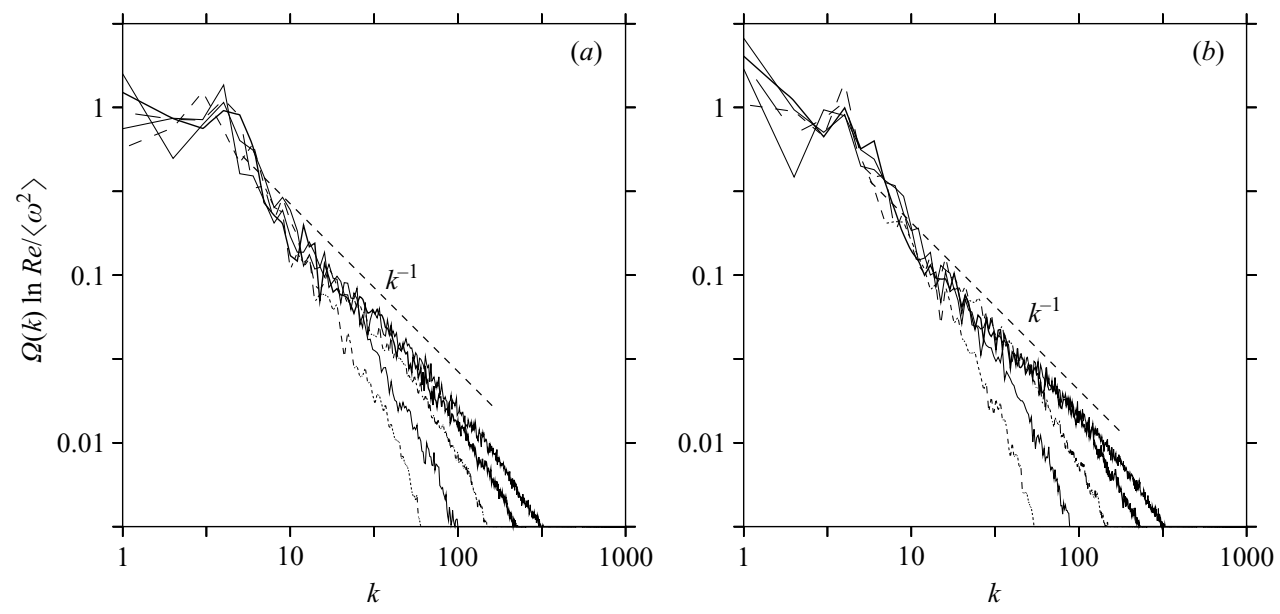

FIGURE 10. Scaled enstrophy spectra, $\Omega(k) \ln R e /\left\langle\omega^{2}\right\rangle$, at the peak enstrophy dissipation time (a) and at the final time (b), for $n=512$ (short dashed), $n=1024$ (thin), $n=2048$ (long dashed), $n=4096$ (bold), and $n=8192$ (thin). A reference slope of $k^{-1}$ is shown as a thick dashed line.

So, we propose just this: keep the form of Batchelor's scaling theory, but use only the inviscid invariants of energy and enstrophy along with $v$ to set $C=\left\langle\omega^{2}\right\rangle / \ln R e$, $k_{v}=\left\langle\omega^{2}\right\rangle^{1 / 4} / \nu^{1 / 2}$ and $k_{0}=\left\langle\omega^{2}\right\rangle^{1 / 2} /\left\langle|\nabla \psi|^{2}\right\rangle^{1 / 2}$ (here with a time-dependent Reynolds number $\left.\operatorname{Re}(t) \equiv k_{\nu}^{2} / k_{0}^{2}\right)$. How does this work in practice? Figure 10 shows the scaled enstrophy spectra $\Omega(k) \ln R e /\left\langle\omega^{2}\right\rangle$ at two characteristic times for the five resolutions. The curves collapse together over the common inertial ranges, and moreover are closely comparable across the two times. There are no adjustable parameters. This demonstrates that the proposed scaling is sensible.

Physically, the proposed form of the enstrophy spectrum, $\left\langle\omega^{2}\right\rangle k^{-1}(\ln R e)^{-1}$, reflects the observation that a fixed amount of enstrophy must spread itself ever more thinly across a widening inertial range as the Reynolds number grows. Note that, although the enstrophy is spread thinly over a wide range of wavenumbers, the energy is dominated by wavenumbers of $O\left(k_{0}\right)$ and smaller. Over these wavenumbers, there remains a finite, non-zero amount of enstrophy, even though it is a negligible proportion of the total at large $R e$.

Our interpretation of this result is that dissipation is playing an increasingly benign role as $R e \rightarrow \infty$. In other words, perfectly inviscid turbulence governed by the Euler equations would eventually exhibit similar behaviour if it were observed down to a fine scale, say $\delta$. Then, we may associate $k_{v}$ with $1 / \delta$.

Finally, we do not suggest that Batchelor's scaling theory persists for all times beyond the peak dissipation of enstrophy, but only for times shortly after it. At much later times, an increasingly dilute population of vortices may come to dominate, leading to steeper spectra in at least the upper part of the inertial range (see Benzi, Patarnello \& Santangelo, 1988 and Santangelo, Benzi \& Legras, 1989).

We are grateful for the wide-ranging e-mail correspondence from many colleagues.

\section{REFERENCES}

Bartello, P. \& Warn, T. 1996 Self-similarity of decaying two-dimensional turbulence. J. Fluid Mech. 326, 357-372. 
BAtCheloR, G. K. 1969 Computation of the energy spectrum in homogeneous two-dimensional turbulence. Phys. Fluids 12, 233-239.

Benzi, R., Patarnello, S. \& Santangelo, P. 1988 Self-similar coherent structures in two-dimensional decaying turbulence. J. Phys. A: Math. Gen. 21, 1221-1237.

Chasnov, J. R. 1997 On the decay of two-dimensional homogeneous turbulence. Phys. Fluids 9, $171-180$.

Davidson, P. A. 2004 Turbulence: An Introduction for Scientists and Engineers. Oxford University Press.

DiPerna, R. J. \& Lions, P.-L. 1989 Ordinary differential equations, transport theory and Sobolev spaces. Invent. Math. 98, 511-547.

DiPerna, R. J. \& MAJDA, A. J. 1987 Concentrations in regularizations for 2-D incompressible flow. Commun. Pure Appl. Maths 40, 301-345.

DMitruk, P. \& Montgomery, D. C. 2005 Numerical study of the decay of enstrophy in a twodimensional Navier-Stokes fluid in the limit of very small viscosities. Phys. Fluids 17, 035114.

DRITSCHEL, D. G. 1989 Contour dynamics and contour surgery: numerical algorithms for extended, high-resolution modelling of vortex dynamics in two-dimensional, inviscid, incompressible flows. Computer Phys. Rep. 10, 77-146.

EyINK, G. L. 2001 Dissipation in turbulence solutions of 2D Euler equations. Nonlinearity 14, 787-802.

Hou, T. Y. \& LI, R. 2006 Dynamic depletion of vortex stretching and non-blowup of the 3-D incompressible Euler equations. J. Nonlinear Sci. 16, 639-664.

Kida, S., Yamada, M. \& OhKitani, K. 1988 The energy spectrum in the universal range of two-dimensional turbulence. Fluid Dyn. Res. 4, 271-301.

Lopes Filho, M. C., Mazzucato, A. L. \& Nussenzveig Lopes, H. J. 2006 Weak solutions, renormalized solutions, and enstrophy defects in 2D turbulence. Arch. Rat. Mech. Anal. 179, 353-387.

Rhines, P. B. 1975 Waves and turbulence on a beta-plane. J. Fluid Mech. 69, 417-443.

Santangelo, P., Benzi, R. \& Legras, B. 1989 The generation of vortices in high-resolution, twodimensional decaying turbulence and the influence of initial conditions on the breaking of self-similarity. Phys. Fluids A 1, 1027-1034.

Tran, C. V. \& Shepherd, T. G. 2002 Constraints on the spectral distribution of energy and enstrophy dissipation in forced two-dimensional turbulence. Physica D 165, 199-212.

Tran, C. V. \& Dritschel, D. G. 2006 Vanishing enstrophy dissipation in two-dimensional NavierStokes turbulence in the inviscid limit. J. Fluid Mech. 559, 107-116.

Zabusky, N. J., Hughes, M. H. \& Roberts, K. V. 1979 Contour dynamics for the Euler equations in 2 dimensions. J. Comput. Phys. 30, 96-106. 